\title{
Probing spin glasses with heuristic optimization algorithms
}

\author{
Olivier C. Martin \\ LPTMS, Université Paris-Sud, \\ Orsay Cedex 91405, France
}

\begin{abstract}
A sketch of the chapter appearing under the same heading in the book "New Optimization Algorithms in Physics" [1] is given. After a general introduction to spin glasses, important aspects of heuristic algorithms for tackling these systems are covered. Some open problems that one can hope to resolve in the next few years are then considered.

PACS numbers: 02.60.Pn Numerical optimization; 75.10.Nr Spin-glass and other random models
\end{abstract}

\section{INTRODUCTION}

Understanding the physical properties of spin-glass materials is a long-standing challenge [2, 3, 4]. Their exotic properties are believed to be controlled by the low energy configurations, a configuration being the specification of the orientation of each of the system's magnetic dipoles. The physical origin of these dipoles is the "spin" of the electrons as in ordinary magnetism, but it is the "irregular" nature of the couplings between these spins that gives these materials their striking properties. To understand most of the phenomenology of equilibrium spin glasses, it should be enough to find the system's ground state and low energy excitations: that is why much effort has focused recently on applying optimization algorithms to spin glasses. Furthermore, spin glasses are the archetypes of complex systems; what is learned here is expected to have a strong impact on our understanding of many other systems with competing effects.

In one of the first attempts to theoretically tackle the competing interactions in these systems, Edwards and Anderson [5] (EA) considered spins on a regular square or cubic lattice, and introduced spin-spin couplings $J_{i j}$ as quenched independent random variables. The corresponding Hamiltonian is

$$
H_{\mathrm{EA}}=-\sum_{\langle i j\rangle} J_{i j} S_{i} S_{j}
$$

They argued that the orientations of the spins $S_{i}= \pm 1$ should "order" at low temperatures, but at random, as would follow for instance from minimizing $H_{\mathrm{EA}}$. Much of the theoretical research on spin glasses since 6] has confirmed this idea, but it has been difficult to make firm claims accepted by all, to a large extent because numerical computations are restricted to small sizes and theoretical approximations are of limited reliability. Work in this field has been quite fruitful in the last 10 years; further progress may be expected but it will require major advances in the algorithmics. We thus consider now some of the corresponding issues.

\section{USING HEURISTIC ALGORITHMS}

It is convenient to divide algorithms for finding ground states into two classes: "exact" or complete, and "heuristic" or incomplete. In the first class, the algorithms will terminate and for sure provide the minimum energy solution; unfortunately they tend to be slow. Our focus here is on heuristic algorithms: such algorithms provide good but not necessarily optimal solutions. They should be used to find ground states only if one can measure their reliability, i.e., if can one convince oneself that the failures to find the true optima arise so rarely that they make no difference for the study. Given this drawback, heuristics have the advantage of being easy to program and of being very fast; this often allows researchers to quickly tackle relatively large systems with little pain.

If the Hamiltonian's ground state is unknown in general, how do we measure the reliability of a heuristic algorithm? We can perform a self-consistent test by running independent multiple starts of the algorithm. As an illustration, assume one has performed 10 independent runs of the algorithm. If all 10 outputs are identical, there is good reason to expect that the true ground state has been found. If on the contrary the 10 outputs do not all agree, the heuristic is not so reliable. Generally, it seems unavoidable for heuristics to become unreliable as the number $N$ of spins becomes large; the challenge is to postpone this bad behavior as much as possible. To illustrate this point, we ran [7] a heuristic called Kernighan-Lin [8] on Ising spin-glass samples of increasing $N$. Fig. 1] shows the distributions of the energies per spin found when using random starts: for each size $(N=20,50,100$ and 170), the histogram is for a single sample, i.e., a single choice of the quenched disorder (the $J_{i j}$ ).

When $N=20$, the distribution is dominated by the probability of finding the lowest energy $E_{0}$ (which presumably is that of the ground state), $P\left(E_{0}\right) \approx 0.98$; this problem size is thus extremely simple to "solve". Increasing the instance size to $N=50$, the distribution is still peaked at $E_{0}$ but much less so: $P\left(E_{0}\right) \approx 0.35$. Going on to $N=100$, the distribution becomes bimodal. Finally, for $N=170$, the peak at $E_{0}$ has disappeared and the distribution is bell shaped. All in all, as $N$ increases, the quality of the algorithm, as measured for instance by $P\left(E_{0}\right)$, deteriorates very clearly. Any heuristic algo- 

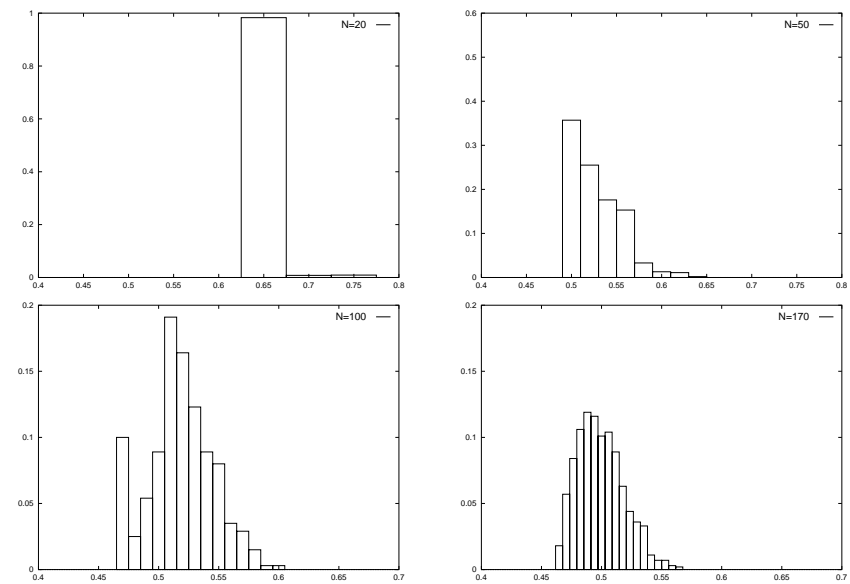

FIG. 1: Distribution of energies per spin for the KL algorithm applied to four samples with $N=20,50,100$, and 170 spins.

rithm will follow this pattern of "simple" to "difficult" as $N$ grows. Such a behavior is generic; the main difference from one heuristic algorithm to another is the typical value of $N$ where there this cross-over from simple to difficult arises.

In practice, a given heuristic will give estimates of $E_{0}$ that are self-averaging, leading to a fixed percentage error in the ground-state energy density in the thermodynamic limit. Furthermore, the probability of finding the exact ground state tends to zero exponentially fast in the number of spins $N$. These properties [7] allow one to compare different heuristics and determine the usefulness of resorting to multiple independent starts. Today's most used algorithms find ground states with a high reliability for 1000 spins but fail badly beyond 3000 spins.

\section{SOME PHYSICAL CHALLENGES}

Let's focus on the EA model in dimension 3 as the stakes there are the highest. The definition of this model is very compact (cf. Eq. 1); it thus seems unbelievable that even the qualitative features of this system are still subject to debate! Of interest to us here are the unsettled issues directly related to optimization, namely the nature of the ground state and its low-energy excitations. In the last few years, several teams [9, 10, 11, 12, 13], each with their own codes, have investigated these issues that we now highlight.

Phase diagram - In most studies, the $J_{i j}$ in Eq. (1) are taken from a distribution symmetric about 0 . If instead $J_{i j}<0$ for only a small fraction of the couplings, the system is ferromagnetic and resembles the ordinary Ising model. As the fraction of anti-ferromagnetic couplings is increased beyond a critical value, the ferro-magnetism disappears. Does the spontaneous magnetization vanish just as the spin-glass ordering appears? Can there be a "mixed" phase where the two types of orderings coexist? Such a phenomenon is expected within the mean- field picture 2]. On the contrary, the droplet/scaling picture [14, 15] says no such coexistence can arise. Only recently has this question been considered numerically but the issue is still far from settled.

An analogous situation appears when an external perturbation in the form of a magnetic field $B$ is applied. In the droplet/scaling picture, the spin-glass ordering is destroyed as soon as $B \neq 0$, leading to a paramagnetic phase. On the contrary, in the mean-field picture, spinglass ordering co-exists with the net magnetization induced by $B$ as long as $B$ is not too large. Numerical studies suggest that no co-existence occurs in 3 dimensions, but further work is necessary.

Energy landscapes - Now consider the organization of the low-energy configurations. Can one give a statistical description whereby some of their properties "scale"? By that we mean that for instance a characteristic energy has a power-law scaling with $N$ as $N \rightarrow \infty$. ( $N$ is the number of spins in the system.) The mean-field picture predicts the following properties.

(i) Clustering: Given a low energy configuration, it is possible to further flip 1, 2, and more spins if they are carefully chosen without changing substantially the value of the excitation energy. The set of low energy configurations form families or "clusters"; two configurations belong to the same cluster if their Hamming distance is very small compared to $N$.

(ii) Replica symmetry breaking: Among the clusters formed by the low energy configurations, consider the ones in which a finite fraction $x$ of the spins are flipped compared to the ground state. (By finite we mean that $x$ is fixed, $0<x<1$, and then one focuses on the large $N$ limit with that given $x$.) In mean field, the corresponding lowest excitation energy is $O(1)$. Furthermore, the system has continuous replica symmetry breaking, meaning that $x$ can take on values that span at least some subinterval of $[0,1]$.

(iii) Ultrametricity: One defines the distance between two configurations as their Hamming distance divided by $N$. One can also define the distance $d(\alpha, \beta)$ between two clusters of configurations from the mean distance of their respective members. In mean field, it turns out that the low energy clusters are organized hierarchically: each cluster is divided into sub-clusters which are themselves sub-divided... Furthermore, if we think of clusters as being points in an abstract space, that space is ultrametric, i.e., all triangles are isosceles.

It is a major challenge to understand whether these remarkable properties also arise in the 3-dimensional EA model. Of course they may not; for instance the droplet/scaling picture may hold instead. In that picture, scaling laws play a central role but so do "positionspace" properties. At the heart of these is the notion of droplet excitations above the ground state; a droplet is defined on a given scale $\ell$ and around a given spin $S_{0}$ as follows. One considers all connected clusters of spins that are enclosed in the cube of side $2 \ell$ centered on $S_{0}$ but which are not contained in that of side $\ell$; among all 
these potential excitations, the droplet is the one of lowest energy. The authors of the droplet/scaling picture postulate the following properties. (i) Droplet energies grow as a positive power of their volume. (ii) There is no replica symmetry breaking. (iii) The organization of the low energy clusters is not hierarchical; nevertheless, the energy landscape is self-similar, i.e., it is a fractal.

Just as for the phase diagram, studies of energy landscapes have not led to any consensus in spite of much work. The root of this problem may very well be that new theoretical frameworks [16, 17] are necessary.

\section{OUTLOOK}

If spin glasses are so controversial, it is largely because finite-size effects in these systems are not well under- stood. Without such an understanding, it is very difficult to extrapolate to the thermodynamic limit, i.e., $N \rightarrow \infty$. This means that algorithmic improvements have to be important, allowing us to go to significantly further in $N$. Currently, we can perform studies involving about 2000 spins. It would probably be necessary to at least double that to have good enough control over the finite-size corrections and to settle long-standing disputes "beyond reasonable doubt".
[1] New Optimization Algorithms in Physics, edited by A. K. Hartmann and H. Rieger (Wiley-VCH, Berlin, 2004), ISBN 3-527-40406-6, http://www.wileyvch.de/publish/en/books/.

[2] M. Mézard, G. Parisi, and M. A. Virasoro, Spin-Glass Theory and Beyond, Vol. 9 of Lecture Notes in Physics (World Scientific, Singapore, 1987).

[3] K. H. Fischer and J. A. Hertz, Spin-Glasses, Vol. 1 of Cambridge Studies in Magnetism (Cambridge University Press, Cambridge, 1991).

[4] Spin Glasses and Random Fields, edited by A. P. Young (World Scientific, Singapore, 1998).

[5] S. F. Edwards and P. W. Anderson, J. Phys. F: Met. Phys. 5, 965 (1975).

[6] K. Binder and A. P. Young, Rev. Mod. Phys. 58, 801 (1986).

[7] G. R. Schreiber and O. C. Martin, SIAM Journal on Optimization 10(1), 231 (1999).

[8] B. Kernighan and S. Lin, Bell System Technical Journal 49, 291 (1970).
[9] A. K. Hartmann, Europhys. Lett. 40, 429 (1997).

[10] J. Houdayer and O. C. Martin, Phys. Rev. Lett. 83, 1030 (1999).

[11] M. Palassini and A. P. Young, Phys. Rev. Lett. 83, 5126 (1999).

[12] E. Marinari and G. Parisi, Phys. Rev. Lett. 86, 3887 (2001).

[13] M. Palassini, F. Liers, M. Juenger, and A. P. Young, Phys. Rev. B 68, 064413 (2003).

[14] D. S. Fisher and D. A. Huse, Phys. Rev. Lett. 56, 1601 (1986).

[15] A. J. Bray and M. A. Moore, in Heidelberg Colloquium on Glassy Dynamics, Vol. 275 of Lecture Notes in Physics, edited by J. L. van Hemmen and I. Morgenstern (Springer, Berlin, 1986), pp. 121-153.

[16] F. Krzakala and O. C. Martin, Phys. Rev. Lett. 85, 3013 (2000).

[17] M. Palassini and A. P. Young, Phys. Rev. Lett. 85, 3017 (2000). 